\title{
Avaliação da compatibilidade química do epóxi LY 1316 com o fluido refrigerante $R 410 A$
}

\section{Resumo}

Este trabalho teve o intuito de investigar o comportamento de um termorrígido, o epóxi LY 1316, fazendo um levantamento da compatibilidade química desse material para com o gás refrigerante R410a, utilizado atualmente em equipamentos de ar condicionado, e assim criar uma metodologia que permita a avaliação de diferentes materiais poliméricos. Foram simuladas algumas das condições de operação às quais o polímero estaria submetido caso fosse aplicado nesse sistema por meio da imersão das amostras em R410a líquido por períodos de catorze dias, de acordo com a norma ASHRAE 97, e então avaliados os efeitos dessa imersão em suas propriedades mecânicas e térmicas. Um grupo de controle foi criado para se avaliar possíveis efeitos da temperatura e diferenciar dos efeitos de ação do refrigerante. Não se observou variações significativas nas análises de DSC, TGA, FTIR e nos ensaios de tração em nenhum dos casos, verificando assim a compatibilidade química entre o epóxi e o refrigerante R410a.

Palavras-chave: Compatibilidade química; Condicionamento de ar; Termorrígido.

\section{Evaluation of chemical compatibility of epoxy LY 1316 with refrigerant R410A}

\begin{abstract}
This work had the main goal of investigating the behavior of a thermoset polymer, epoxy LY 1316, making a survey of the chemical compatibility of this material in the refrigerant fluid R410a, currently used in air conditioning equipment, so creating a methodology that evaluates different polymers. The operating conditions of the system were simulated by immersing samples in liquid R410a for a period of fourteen days, according to the ASHRAE 97 standard, then the effects of this immersion on their mechanical and thermal properties were evaluated. A control group was created in order to evaluate possible effects of temperature, and to differentiate them from the effects of the refrigerant. No significant variations were observed in the analyzes of DSC, TGA, FTIR and on tensile tests for all samples, thus concluding chemical compatibility between the epoxy and the R410a refrigerant.
\end{abstract}

Keywords: Chemical compatibility; Air conditioning; Thermoset.

\section{Introdução}

Sistemas de refrigeração e condicionamento de ar são amplamente utilizados para diversos fins, seja na indústria, em câmaras frias para o armazenamento de alimentos, ou em residências, melhorando o conforto térmico das pessoas [1]. Acordos entre países como o estabelecido no Protocolo de Montreal buscam eliminar completamente o uso de HCFCs como o Clorodifluorometano (R22) até 2040. Em substituição ao R22, os aparelhos novos são projetados para serem usados com o gás refrigerante $\mathrm{R} 410 \mathrm{a}$, uma mistura de difluorometano e pentafluoretano, que não possui potencial de destruição da camada de ozônio [2].

Sistemas Split, que tem se popularizado nos últimos anos, são constituídos por duas unidades, uma interna, que contém a válvula de expansão e o evaporador, e outra externa,

1Programa de Pós-graduação em Ciência e Engenharia de Materiais, Departamento de Engenharia Mecânica, Universidade do Estado de Santa Catarina, Joinville, SC, Brasil.

*Autor correspondente: petrius_tambosi@hotmail.com 
que contém o compressor e o condensador. As unidades são conectadas por dois tubos de cobre com isolamento térmico, que transportam o fluido refrigerante. Essas tubulações são utilizadas por apresentarem grande flexibilidade, que aliadas com a resistência mecânica e impermeabilidade aos gases garantem grande vida útil ao conjunto.

Tubulações de cobre, no entanto, possuem custo elevado, se comparadas a tubulações poliméricas. São poucos os estudos envolvendo análises de compatibilidade química entre materiais poliméricos e refrigerantes, e certamente menos estudos existem com o R410a. Cavestri [3] fez um levantamento da compatibilidade de diversos materiais poliméricos com refrigerantes como o R22. Em suas análises, constatou-se que as principais alterações sobre as propriedades dos polímeros foram em maior nível devido à influência da temperatura do que da ação do fluido refrigerante. Seus métodos de análise formaram parte da base deste estudo.

Tendo em vista a lacuna de conhecimento e informações a respeito da degradação ou compatibilidade de materiais poliméricos com fluidos refrigerantes, bem como o potencial para aplicação no mercado dos mesmos, foi feito o desenvolvimento deste estudo para avaliar o comportamento de um epóxi em sistemas de condicionamento de ar e gerar um roteiro que possibilite análises similares com outros materiais poliméricos.

\section{Metodologia}

\subsection{Materiais}

Foi utilizada a resina Araldite ${ }^{\circledR}$ LY 1316-2 BR com o endurecedor Aradur ${ }^{\circledR}$ HY 951 - em proporções de 100 para 13 partes, respectivamente - e o desaerante A525 da Huntsman, na proporção de $0,5 \%$ da massa total, com a finalidade de auxiliar a remover as bolhas de ar geradas na agitação da mistura.

O fluido refrigerante utilizado foi o Suva ${ }^{\circledR} 410 \mathrm{~A}$ (R410a), fabricado pela DuPont, fornecido em cilindros de $11,35 \mathrm{~kg}$. Para a purga do sistema, entre as aplicações de vácuo, foi utilizado o Argônio (Ar), com pureza de 99,9\%, por ser um gás inerte.

\subsection{Condições de contorno}

Foram simuladas condições próximas às de operação do fluido refrigerante num equipamento de ar condicionado nos pontos de condução desse fluido de uma unidade à outra do Split. O ponto mais crítico para o sistema é o presente na entrada da unidade condensadora, em que a temperatura atinge $50{ }^{\circ} \mathrm{C}$, a uma pressão de cerca de 30 bar, de acordo com o diagrama de Mollier do fluido refrigerante R410a [2], condição adotada para a análise de compatibilidade.

\subsection{Corpos de prova de Epóxi}

O endurecedor e seguidamente o desaerante foram adicionados à resina epóxi segundo as recomendações do fabricante pelo método de casting, consistindo em mistura manual dos componentes em um becker com a utilização de um bastão de vidro por 15 minutos e cerca de 60rpm. Após a mistura despejou-se a resina em moldes de silicone no formato das amostras utilizadas.

Para ensaios de tração, as amostras foram fabricadas no formato de corpos de prova (CDP) de tração do Tipo I, segundo os parâmetros da norma ASTM D638 - 14 [4]. A retirada dos corpos de prova dos moldes foi feita após um período de $24 \mathrm{~h}$ de cura. Foi então feita uma pós-cura do material em estufa por $4 \mathrm{~h}$ a $60{ }^{\circ} \mathrm{C}$.

\subsection{Ensaio de compatibilidade química}

O estudo de compatibilidade química teve como base a norma ANSI/ASHRAE 97 [5]. Para tal, com base também no projeto de Cavestri [3] os autores desenvolveram um equipamento que permitisse que os CDP ficassem imersos em fluido refrigerante em temperatura controlada - banho térmico - de $50{ }^{\circ} \mathrm{C}$ por um período de 14 dias. O cilindro em que os CDP são armazenados, bem como o equipamento e o esquema com seus componentes são apresentados na Figura 1a-c, espectivamente.

Como é possível observar na Figura 1, o sistema foi projetado de modo a permitir a evacuação e purga completa e de ser alimentado com fluido no estado líquido, mantendo-se as proporções de massa da mistura azeotrópica do R410a [6]. Os cilindros de armazenamento podem ser facilmente conectados e removidos do sistema, permitindo que diversas amostras sejam submetidas simultaneamente ao banho térmico de 14 dias. Para se certificar que os CDP estarão imersos em uma quantidade suficiente de fluido refrigerante, foi feita a medição da massa dos cilindros com CDP antes e depois de serem evacuados e alimentados com R410a, e ao fim do período de imersão, para se certificar da estanqueidade do cilindro.

Foram elaborados três grupos de amostras. O primeiro, para ser utilizado como referência do material, refere-se a amostras de epóxi logo após o procedimento de pós-cura, exposto apenas à temperatura e pressão ambiente por $24 \mathrm{~h}$. O segundo, de modo a se observar uma possível influência da exposição à temperatura, como verificado por Cavestri [3], consiste de amostras submetidas à temperatura da condição de contorno por um período de 14 dias, porém em atmosfera neutra. O terceiro grupo é constituído por amostras imersas em fluido refrigerante por 14 dias à temperatura e pressão das condições de contorno, descritas neste trabalho. Esses grupos de amostras são aqui denominados Epóxi, Temperatura e R410a, respectivamente.

\subsection{Ensaio de resistência à tração}

As amostras foram testadas em uma máquina de ensaios universais Oswaldo Filizola, modelo AME-5KN, do laboratório da UDESC-CCT, com uma célula de carga FLINTEC ULB de $500 \mathrm{~kg}$. Foi utilizada uma velocidade de ensaio de $5 \mathrm{~mm} / \mathrm{min}$, como recomendado pela ASTM D638 - 14 para ensaios de tração com materiais termorrígidos, obtendo-se a tensão máxima 


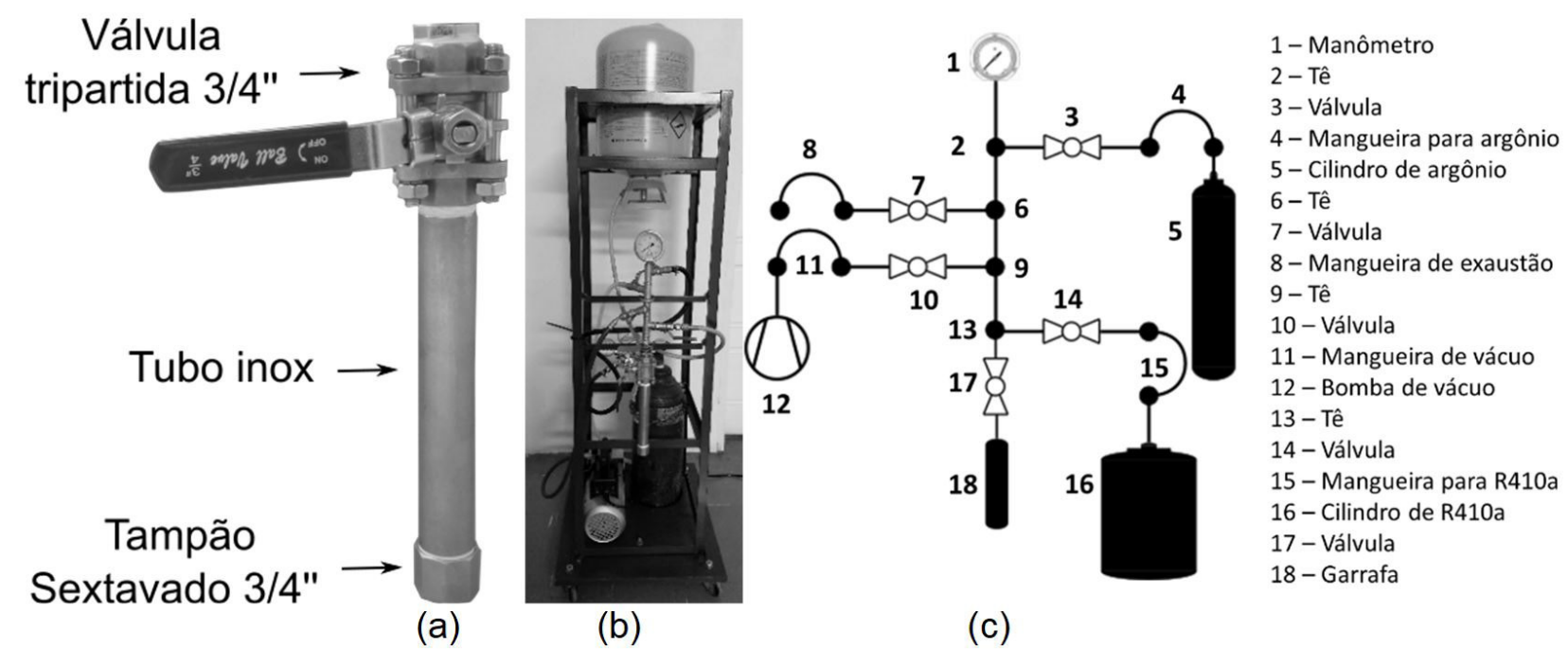

Figura 1. Cilindro (a), equipamento (b) e esquema com componentes (c) para imersão de amostras de polímeros em fluido refrigerante.

e o módulo de elasticidade. A distância entre as garras era de $63 \mathrm{~mm}$, e não se utilizou extensômetro.

\subsection{Espectroscopia no infravermelho com Transformada de Fourier - FTIR}

Utilizou-se um espectrofotômetro da marca PerkinElmer, modelo Spectrum 100 Series, do laboratório da empresa parceira. As amostras foram cortadas e usou-se o modo ATR (análise superficial), com números de onda de $3800 \mathrm{a} 600 \mathrm{~cm}^{-1}$.

\subsection{Calorimetria Exploratória Diferencial - DSC}

As análises foram feitas em um equipamento de DSC modelo Q20, da TA Instruments, da empresa parceira. Fez-se um aquecimento em atmosfera de nitrogênio, de 20 a $220^{\circ} \mathrm{C}$, a uma taxa de $10^{\circ} \mathrm{C} / \mathrm{min}$, com o objetivo de acompanhar a eventual eliminação de fluido refrigerante retido no material e alterações devido a efeitos associados a pós-cura. As amostras foram geradas por raspagem, e o pó obtido colocado em cadinhos de alumínio, ficando com cerca de $4 \mathrm{mg}$ em cada.

\subsection{Análise Termogravimétrica - TGA}

As análises termogravimétricas foram realizadas em um equipamento de TGA modelo STA 449C, da NETZSCH, do laboratório da UDESC. Realizou-se um aquecimento de 20 a $850^{\circ} \mathrm{C}$, a uma taxa de $10^{\circ} \mathrm{C} / \mathrm{min}$, em atmosfera de nitrogênio. As amostras foram geradas por raspagem, e cerca de $20 \mathrm{mg}$ do pó obtido foram colocados em cadinhos de alumínio.

\section{Resultados}

A Tabela 1 e a Figura 2 apresentam os valores médios obtidos para a tensão máxima e módulo de elasticidade dos grupos de amostras analisados.
As curvas obtidas para os ensaios de FTIR são apresentadas na Figura 3.

As análises térmicas para as amostras de epóxi nas três condições avaliadas são apresentadas na Figura 4. A Figura $4 \mathrm{a}$ exibe a TGA e Figura $4 \mathrm{~b}$ as curvas de DSC.

\section{Discussão}

\subsection{Ensaio de tração}

Segundo o manual da fabricante Huntsman [7], o esperado para a resistência à tração é de 49 a 52MPa. Os valores obtidos neste estudo, como observados na Tabela 1 e Figura 2, encontram-se próximos do esperado pelo fabricante, considerando-se o desvio-padrão. Também, se considerando o desvio-padrão, não se observa uma diferença significativa nos resultados dos três grupos de amostras estudados, indicando não haver influência da exposição à temperatura ou ao fluido refrigerante sobre as propriedades do epóxi.

Fluidos refrigerantes devem atender a certos critérios de segurança para que possam ser comercializados. Entre eles, a característica essencial que mais se destaca é a necessidade de serem quimicamente estáveis e inertes, especialmente com os materiais tipicamente utilizados em sistemas de refrigeração [8]. Essa estabilidade química, somada com a boa resistência química dos epóxis a solventes [9], acaba sendo evidenciada na aparente não alteração das propriedades após o contato e imersão do epóxi em fluido refrigerante.

\subsection{FTIR}

Os três grupos de amostras foram analisados em FTIR, a fim de observar diferenças no comportamento de transmitância de luz, o que poderia indicar uma possível retenção, absorção ou adsorção do fluido refrigerante pela resina epóxi ou então um efeito sobre o nível de cura ou 
Tabela 1. Tensão e Módulo de elasticidade para os grupos de amostras de epóxi

\begin{tabular}{lcccc}
\hline \multicolumn{1}{c}{ Tipo } & Tensão (MPa) & Desvio-Padrão (MPa) & Módulo de Elasticidade (GPa) & Desvio-Padrão (GPa) \\
\hline Epóxi & 48,21 & 3,22 & 2,57 & 0,12 \\
14 dias & 48,90 & 2,08 & 2,59 & 0,15 \\
R410a & 47,27 & 2,50 & 2,51 & 0,10 \\
\hline
\end{tabular}

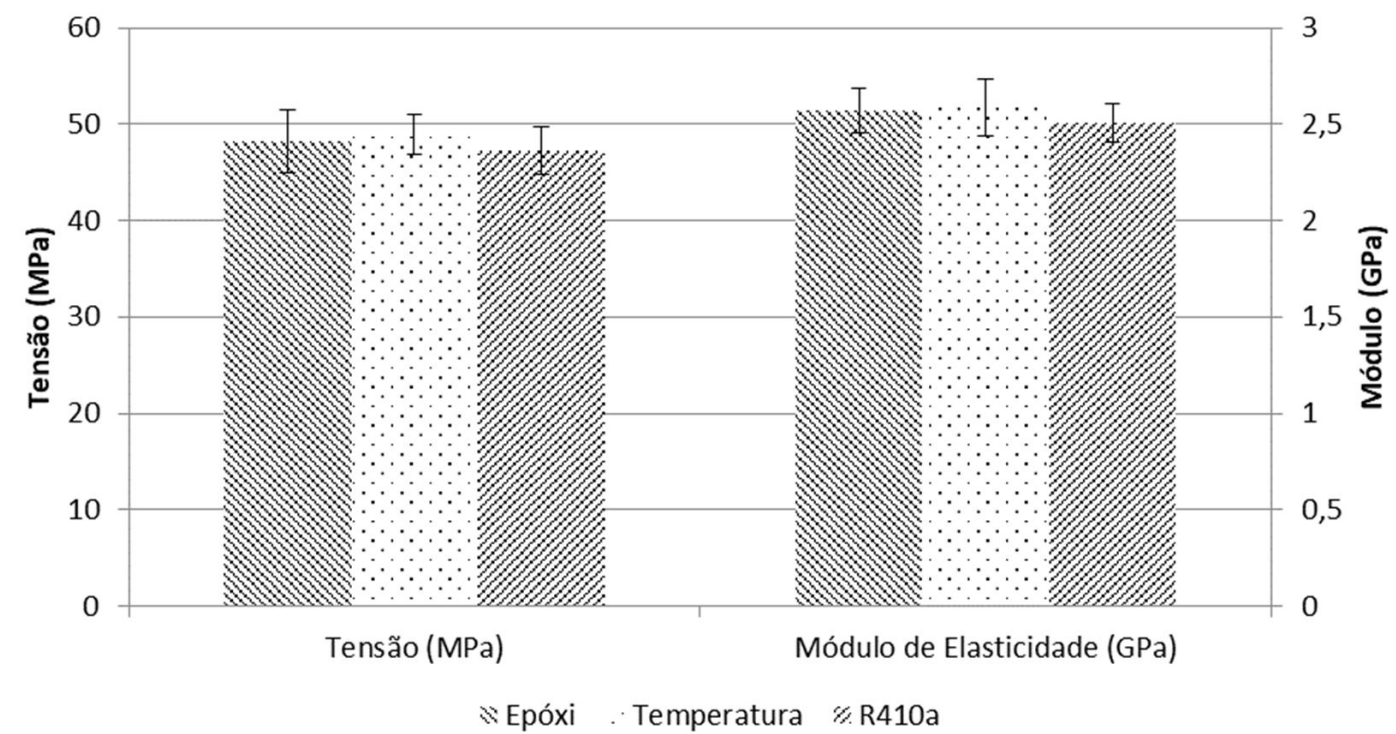

Figura 2. Tensão e Módulo de elasticidade para os grupos de amostras de epóxi.

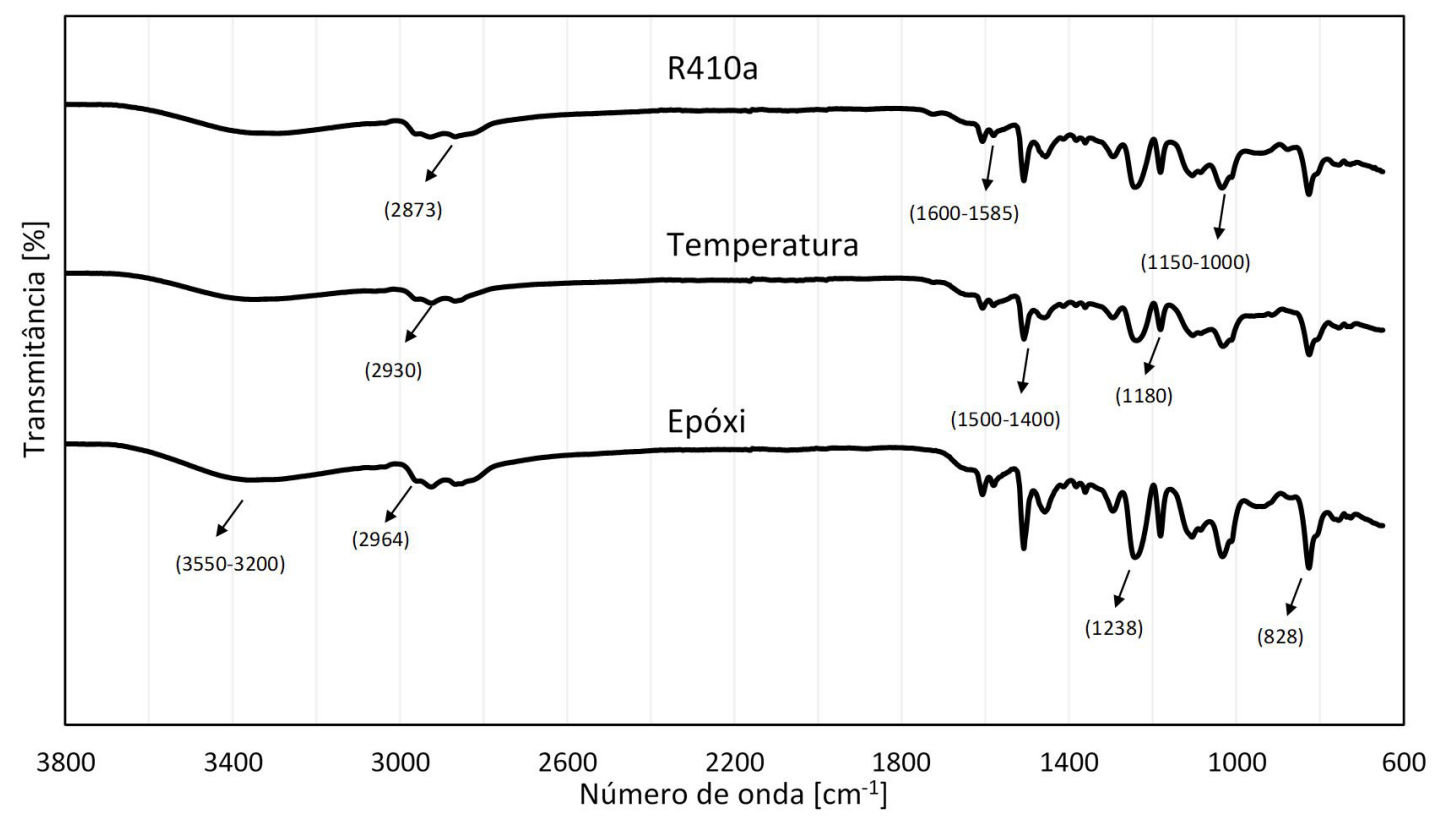

Figura 3. FTIR obtido para as amostras de epóxi.

degradação da resina devido à exposição à temperatura pelo período. O levantamento dos principais picos foi feito de acordo com as análises de Farias [10], Silva [11], para resina LY 1316, e com base no material de Kuptsov e Zhizhin [12].

É possível observar na Figura 3 uma faixa de absorção para o grupo hidroxila, $\mathrm{O}-\mathrm{H}$, em estiramento, na região de 3550 a $3200 \mathrm{~cm}^{-1}$. Na região de 3000 a $2800 \mathrm{~cm}^{-1}$, verifica-se estiramento de $\mathrm{C}-\mathrm{H}$ alifático de grupos $\mathrm{CH}, \mathrm{CH}_{2}$ e $\mathrm{CH}_{3}$. O grupo $-\mathrm{CH}_{3}$ aparece em $2934 \mathrm{~cm}^{-1}$ em estiramento assimétrico e em $2860 \mathrm{~cm}^{-1}$ em estiramento simétrico. Destaca-se um pico de absorção em $1508 \mathrm{~cm}^{-1}$, associado à ligação $\mathrm{C}=\mathrm{C}$ do anel aromático em estiramento simétrico, que também pode ser observado em picos na região de 1600 a $1585 \mathrm{~cm}^{-1}$. O grupo $\mathrm{C}-\mathrm{CO}-\mathrm{C}$ em estiramento aparece 


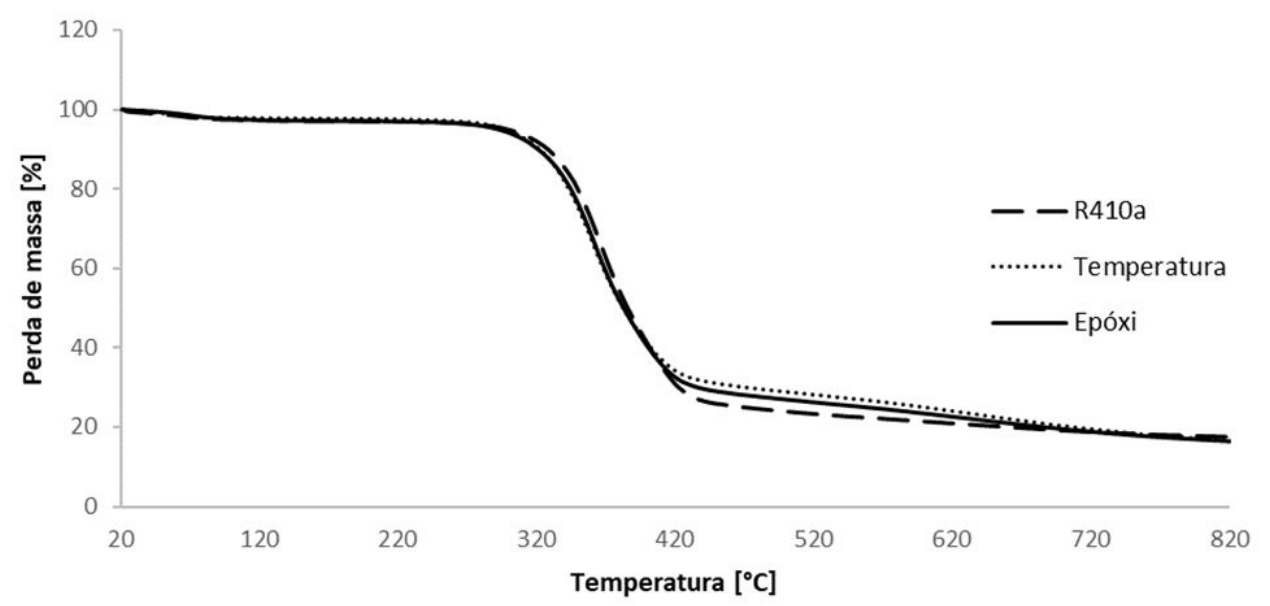

(a)

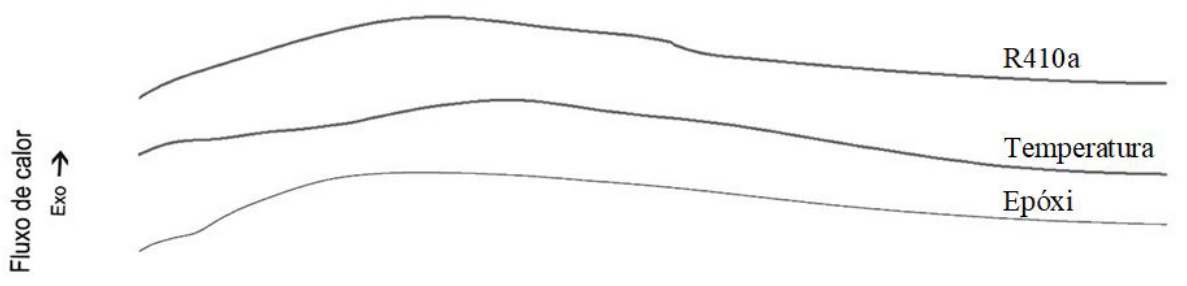

(b)

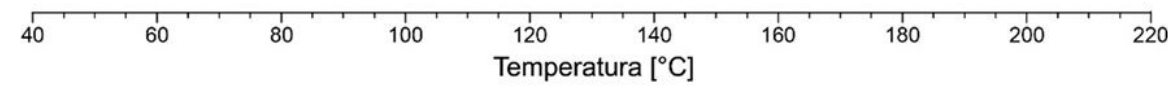

Figura 4. (a) TGA e (b) DSC das amostras de epóxi.

próximo a $1180 \mathrm{~cm}^{-1}$. O éter $\mathrm{C}-\mathrm{O}-\mathrm{C}$ aparece em estiramento assimétrico em $1030 \mathrm{~cm}^{-1}$, região de $1150-1000 \mathrm{~cm}^{-1}$.

Outros dois picos característicos do epóxi são evidenciados pela absorção observada nos números de onda 1238 e $828 \mathrm{~cm}^{-1}$. Ambos são devidos à presença do anel epóxi, em estiramento simétrico no primeiro caso e estiramento assimétrico no segundo.

Verifica-se que as curvas dos três grupos de amostras evidenciam aspectos bastante similares, não sendo observadas alterações sensíveis no comportamento térmico do material em seu aspecto original para as demais condições. A absorção no intervalo de 950 a $810 \mathrm{~cm}^{-1}$, que representa o anel epóxi, permite também avaliar o grau de cura do material. Como não se observou uma variação significativa nessa região, pode-se concluir que a rota utilizada para produção das amostras (cura por $24 \mathrm{~h}$ e pós cura por $4 \mathrm{~h} \mathrm{a} 60^{\circ} \mathrm{C}$ ) permitiu atingir um alto grau de cura, e que nem o efeito da temperatura nem a exposição ao fluido refrigerante alterou notavelmente esse teor.

\subsection{TGA e DSC}

A partir da Figura 4a, pela interseção das tangentes de uma curva é possível determinar a temperatura de Onset $\left(\mathrm{T}_{\text {onset }}\right)$, obtendo-se $325^{\circ} \mathrm{C}$. O ponto de inflexão, em $380^{\circ} \mathrm{C}$, foi observado com base na curva de Análise térmica diferencial (DTA), que também revelou uma temperatura de pico $\left(\mathrm{T}_{\text {pico }}\right)$ de $345^{\circ} \mathrm{C}$, temperatura na qual se registra a perda de massa mais acentuada, aparecendo como um pico na curva da primeira derivada (DTA).

Um comportamento semelhante para a curva do epóxi LY 1316 foi observado por Farias [10], Silva [11] e Backes [13]. As curvas obtidas nesse estudo para os diferentes grupos de amostras apresentam aspectos bastante similares, como nas análises anteriores - de tração e FTIR - não sendo observadas alterações sensíveis no comportamento térmico do material em seu aspecto original para as demais condições, com um estágio principal de degradação e perda de massa. Muitos fatores podem influenciar no comportamento da curva de TGA, de acordo com Canevarolo [14], entre eles o tamanho e a granulometria da massa da amostra, que pode ser um dos motivos para a pequena anomalia térmica entre a região de $430^{\circ} \mathrm{Ce} 680^{\circ} \mathrm{C}$. Apesar desta pequena anomalia, os valores para Temperatura de inflexão e $\mathrm{T}_{\text {onset }}$ permaneceram similares, bem como a massa residual percentual após a degradação, restando cerca de $17 \%$ em $800{ }^{\circ} \mathrm{C}$.

$\mathrm{Na}$ análise de DSC, exibida na Figura 4b, não foi possível observar transições de primeira ordem, isto é, picos exotérmicos ou endotérmicos que poderiam indicar perda de massa ou dessorção (nesse caso, para o fluido refrigerante), cristalização ou reações de polimerização e cura [14]. Backes [13] em seu estudo levantou as curvas de DSC para amostras epóxi LY 1316 com e sem pós cura, 
verificando indícios de cura residual identificados através de um pico exotérmico, o que não aconteceu com as amostras que passaram pelo processo de pós cura, de modo similar ao comportamento observado por todas as amostras deste estudo. É importante salientar que não há qualquer pico endotérmico referente a relaxações entálpicas, uma vez que estas ocorreram durante a pós-cura, como também observado por Backes [13]. Além do observado na análise de FTIR, este é um bom indicativo de que o processo de pós-cura utilizado na metodologia deste trabalho foi efetivo para que se atingisse um elevado grau de cura do material.

\section{Conclusão}

O equipamento de compatibilidade química desenvolvido pelos autores mostrou-se adequado para o estudo com o fluido refrigerante R410a, suportando a pressão exercida pelo gás durante todo o período de imersão sem perdas significativas de massa de fluido, criando um ambiente adequado - estanque e isolado da interferência externa - para comportar as amostras poliméricas, conforme as normas internacionais. Apresentou-se com fácil operação, respeitando todas as condições de projeto e permitindo o ensaio simultâneo de diversas amostras poliméricas.

A rota implementada para a verificação de compatibilidade química mostrou-se adequada para a verificação de alterações nas propriedades do epóxi LY 1316. Os três grupos estudados - a resina em seu estado original; a resina submetida à temperatura de ensaio por 14 dias; a resina exposta ao fluido refrigerante e à temperatura de ensaio por
14 dias - não apresentaram diferenças significativas entre si. As boas propriedades de resistência mecânica e química das resinas epóxi, juntamente com a baixa reatividade e alta estabilidade do R410a, foram evidenciadas através da não constatação de alterações significativas nas propriedades mecânicas, espectroscópicas e térmicas.

Os ensaios de tração revelaram uma tensão máxima próxima aos valores esperados pelo fabricante da resina e ao encontrado em outros estudos com a mesma resina, indicando boa qualidade na preparação dos corpos de prova. A análise de FTIR não revelou a presença de novos picos ou faixas de absorção de onda, indicando não haver uma retenção, absorção ou interação das moléculas do fluido refrigerante com as amostras de epóxi. As curvas de TGA tiveram um comportamento muito similar, observando-se as mesmas temperaturas de início de degradação, inflexão e de pico, com uma massa residual ao fim do ensaio de cerca de $17 \%$ para todos os casos. A análise de FTIR e as análises térmicas de TGA e DSC não demonstraram alteração no grau de cura da resina em nenhuma das condições, indicando que a rota utilizada, com cura por $24 \mathrm{~h}$ à temperatura ambiente e pós cura por $4 \mathrm{~h}$ a $60^{\circ} \mathrm{C}$, foi suficiente para que se atingisse um elevado grau de cura da resina epóxi. Assim, verificou-se que o epóxi LY 1316 apresentou compatibilidade química com o refrigerante R410a, tornando-o um candidato quimicamente apto a aplicações que trabalhem com o fluido em condições de operação similares ao ponto crítico de sistemas de ar condicionado do tipo Split $\left(50^{\circ} \mathrm{C}, 30 \mathrm{bar}\right)$. No entanto, estudos adicionais - especialmente de permeabilidade - são necessários antes que este material seja considerado em projetos comerciais.

\section{Referências}

1 Stoecker W, Jones J. Refrigeracao e ar condicionado. São Paulo: McGraw-Hill Do Brasil; 1985.

2. Dupont. DuPont ${ }^{\mathrm{TM}}$ Suva $^{\circledR} 407 \mathrm{C}$ (R-407C) and DuPont ${ }^{\mathrm{TM}}$ Suva $^{\circledR} 410^{\mathrm{a}}$ (R-410A), Delaware, USA: Dupont; 2016.

3. Cavestri RC. Compatibility of Refrigerants and Lubricants with Engineering Plastics. Dublin: Ohio: Air-Conditioning and Refrigeration Technology Institute; 1993.

4 American Society for Testing and Materials. ASTM D638-14: Standard Test Method for Tensile Properties of Plastics. West Conshohocken, PA: ASTM; 2014.

5. American Society of Heating, Refrigerating and Air-Conditioning Engineers. ASHRAE 97-2007: Sealed Glass Tube Method to test the chemical stability of materials for use within refrigerant systems. Georgia: ASHRAE; 2007.

6. Lavelle J. Understanding Refrigerant Blend Performance. RSES Journal. 2006;VOL(ISSUE):29-33.

7. Huntsman. Araldite ${ }^{\circledR}$ Ly 1316 - 2 Br Aradur ${ }^{\circledR}$ Hy 951 Br. Texas: Huntsman; 2013.

8 Mclinden MO, Didion DA. The Search for Alternative Refrigerants -- A Molecular Approach. In: International Refrigeration and Air Conditioning Conference. Paper 69; 1988 July 1; Purdue: Purdue University: 1988. [acesso em 14 de janeiro de 2019]. http://Docs.Lib.Purdue.Edu/Iracc/69

9 Pizzutto CE, Pezzin SH, Coelho LAF, Amico SC. Compósitos Epóxi/Nanotubos de carbono preparados com dispersão de reforço no endurecedor [dissertação]. Joinville: Universidade do Estado de Santa Catarina, Ciência e Engenharia de Materiais; 2008.

10 Farias MAD. Nanocompósitos de Nanotubos de carbono de paredes múltiplas com matrizes híbridas Epóxidocopolissilsesquioxanos [dissertação]. Joinville: Universidade do Estado de Santa Catarina, Ciência e Engenharia de Materiais; 2010. 114 p. 
11 Silva LVD. Efeito da degradação nas propriedades de hastes pultrudadas expostas a ambientes agressivos [tese]. Porto Alegre: Universidade Federal do Rio Grande do Sul; 2017. 145 p. [acesso em 28 jan. 2019]. Disponível em: https://Lume.Ufrgs.Br/Handle/10183/165202

12 Kuptsov AH, Zhizhin GN. Handbook of Fourier Transform Raman and Infrared Spectra of Polymers. Amsterdam, The Netherlands: Elsevier Science; 1998.

13 Backes EH. Desenvolvimento de Nanocompósitos Híbridos de Epóxi/Ntcpm/Cargas Minerais e avaliação das propriedades mecânicas, elétricas e térmicas. [tese]. São Carlos Universidade de Federal de São Carlos, Ciência e Engenharia de Materiais; 2016. 173 p.

14 Canevaloro SV Jr. Ciência dos polímeros: um texto básico para tecnólogos e engenheiros. São Paulo: Artliber; 2002.

Recebido em 08 Fev. 2019

Aceito em: 09 Jun. 2020 